\title{
MIMacrothink
}

\section{Long Range Security and Safety Tracking System for Merchant Vessels}

\author{
Ramzi Zaher \\ MBA Student at Jordanian University \\ Arab Bridge Maritime Company Consultant Engineer \\ Manager Director of Marine Engineering of Electrical and Electronic Systems corp. \\ P.O.Box2346 Aqaba77110- JORDAN \\ E-mail: technical@meeescorp.com
}

Doi:10.5296/ijld.v4i3.5963ＵRL: http://dx.doi.org/10.5296/ijld.v4i3.5963

\begin{abstract}
Seventy percent of the earth's surface is covered by water, in this large area the vessels are trading, fishing and transporting. Whatever the purpose of sailing we can consider it shipping and is relaying and requiring safety navigation, taking into account keeping clean environment. The Shipping plays a vital role in the economics of most countries so the safety and security control and monitoring from shore is significant. This paper will explain the maritime vessels security systems and safety and will be concentrated on the integration of the security system devices, safety system devices and transmission through satellite communication systems to the shore side, by other means main office on land, to raise the level of safety and security. The safety by choice not by chance
\end{abstract}

Keywords: IMO international maritime organization, GIS geographic information system, SOLAS safety of live at sea,VDR voyage data recorder ,ECDIS electronic chart display and information system, RADAR radio detection and ranging, AIS automatic identification system ,MIS marine information system ,PLC programming logic control ,SCADA supervisory control and data acquisition, GPS global position system, GMDSS global maritime distress and safety system ,SASS Ship Security Alert System ,NAVTEX national weather service BNWAS bridge navigational watch alarm system ,NMEA 183 combined electrical and data specification for communication between marine electronics .

\section{Introduction}

Arguably Word shipping tries to develop according to the requirements of trade. The security and safety system of several merchant vessels over the world such as passenger vessel, fishing vessel, bulk carrier, oil tanker and general cargo can be raised by integration system of multiple devices which are installed onboard .The ship manager are there, in position to contribute and interest to safer shipping and cleaner oceans. Monitoring the vessel's security and safety from shore, wherever the vessels are sailing, helps the shipping management to maximize vessel's chances of avoiding pirate attack and capture, control the fuel budget and extra expenditures, increase the vessel performance, manage the crew system, apply the preventing maintenance of the machinery and safety equipments, monitor the vessel root and weather, estimate the vessel arrival and departure time and facilitate the management decision making .To implement idea of the long range safety and security system needs aggregate mandatory security devices in the wheel house and safety device in the main engine department and transmit the compound of data to the another side of the world via the satellite 
communication system to the main control office. Technically and theoretically it is possible ,but budgetary it is costly. Unfortunately very view studies not addressed with this subject profusely and strongly, In my opinion because of the budget problems. In this paper will exploit the mandatory devices onboard in the wheelhouse and the main engine machineries and avoid as much the study allow to add extra devices in order to minimize the cost of the system .Obviously the extra cost will be formulated into satellite communication fees form vessel to the shore. But when the safety and security management matters the cost will not be the substantial impact on the director's decisions. Imagine possible to predict the fault, fire, defect or hidden disaster before happen.

"Whilst the public only hear about piracy and maritime terrorism when there is a serious incident, the problem is continuous and the costs to the industry, and hence to the end consumers of maritime trade, are growing. As in other armed conflicts, technology could provide more reliable, effective deterrents and safer counters. Meeting that challenge is what IET's engineers and technologists can do. Through Insights like this, and by enabling debate, the IET's Marine Transport Group encourages innovation in the search for effective solution" (Brooks, B., 2013)

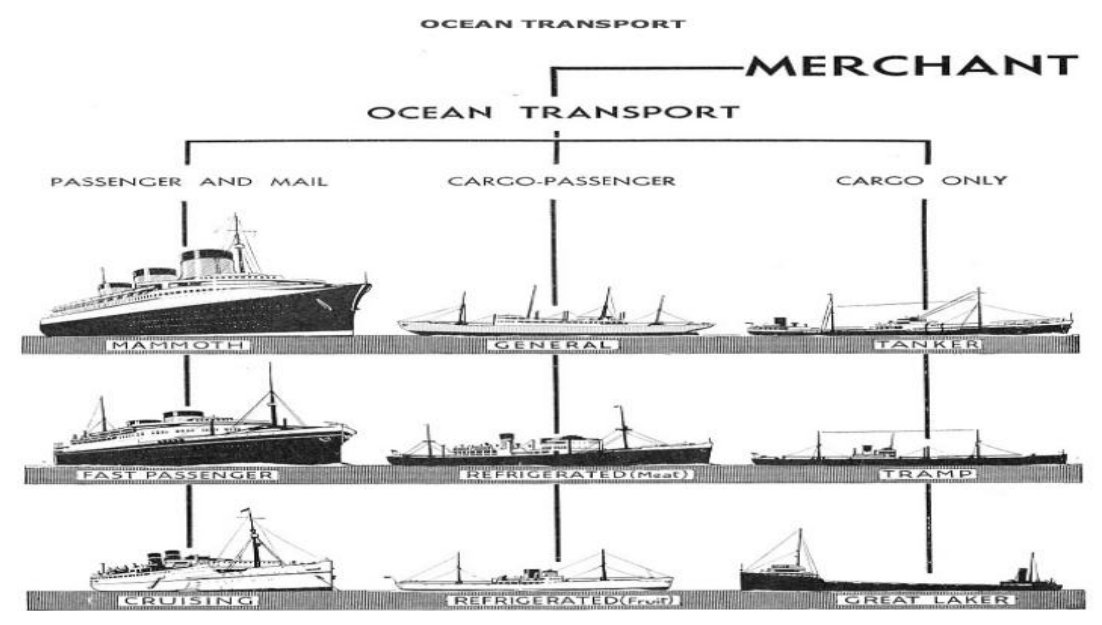

Figure 1.General view of merchant ship types.

\section{Vessel's Fundamentals Sections}

The vessel's departments divided into two essential departments' wheelhouse and main engine. The wheel house where is the navigation equipments installed and wheel steering support to remote control system ,from the wheelhouse the vessel can command via navigation crew department (Captain ,chief officer and other officers ) .Main engine department consist of the vessel's powering engine force, electrical supply and the safety system protect main engines, generators and auxiliary main engine's department all are supervisory by engine crew department (chief engineer, second engineer and other stuff of engineers and oilers ). It is necessarily to monitor and control mandatory devices by alarms systems and pr-warning. In this report will demonstrate each device separately and the possible output signal can be used to interface into one system from each device.

\subsection{Wheelhouse control system illustration}




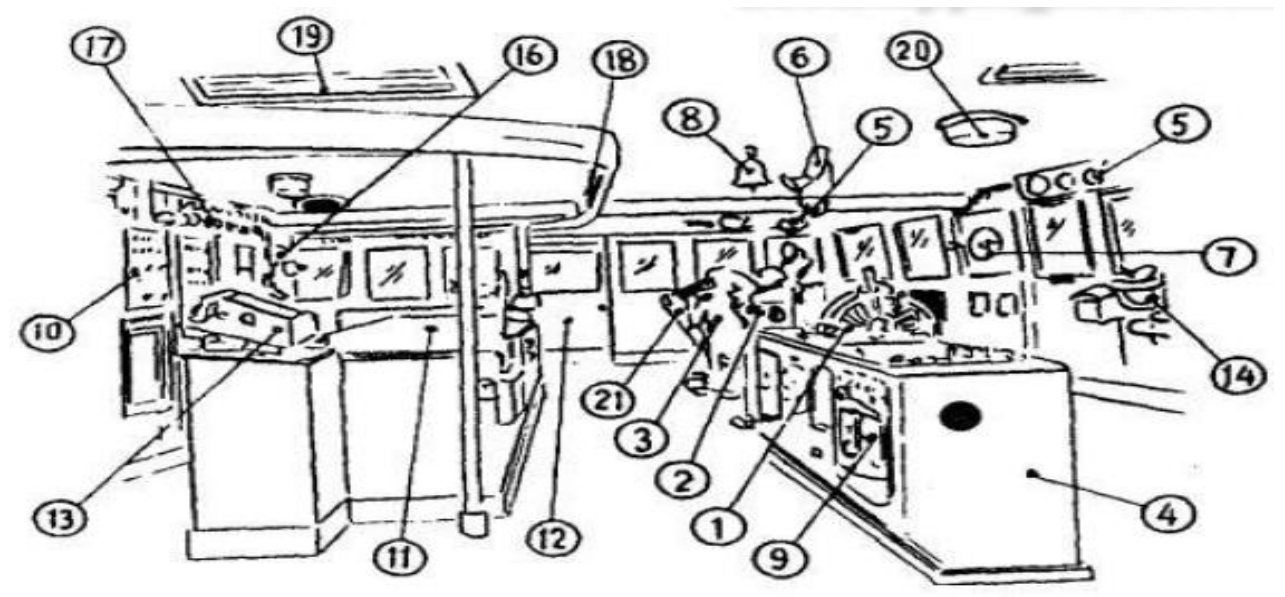

Figure 2: Wheelhouse control system in general.

Table 1. The wheel house control system in general

\begin{tabular}{|c|c|c|c|c|c|c|}
\hline$\overline{\text { No. }}$ & \multicolumn{2}{|c|}{ Description } & No. & & \multicolumn{2}{|c|}{ Description } \\
\hline & 1 & Engine telegraph & & 1 & & Chart table \\
\hline & & & 1 & & & \\
\hline & 2 & Gyro-pilot steering stand & & 1 & & Outside door way \\
\hline & & & 2 & & & \\
\hline & 3 & Radar indicator & & 1 & & Small type \\
\hline & & & 3 & & indicat & \\
\hline & 4 & Bridge console stand & & 1 & & Repeater compass of \\
\hline & & & 4 & & gyro & \\
\hline & 5 & Projector compass & & 1 & & Indicator of $\log$ \\
\hline & & & 5 & & & \\
\hline & $\epsilon$ & Voice tube to flying bridge & & 1 & & Loran receiver indicator \\
\hline & & & 6 & & & \\
\hline & 7 & Clear view screen & & 1 & & Signal flag shelf \\
\hline & & & 7 & & & \\
\hline & $\varepsilon$ & Signal bell & & 1 & & Curtain rail of chart \\
\hline & & & 8 & & room & \\
\hline & c & Telephone & & 1 & & Ventilator duct \\
\hline & & & 9 & & & \\
\hline & 1 & Switch board & & 2 & & Room light \\
\hline 0 & & & 0 & & & \\
\hline
\end{tabular}

\subsection{Engine Compartment Illustration}




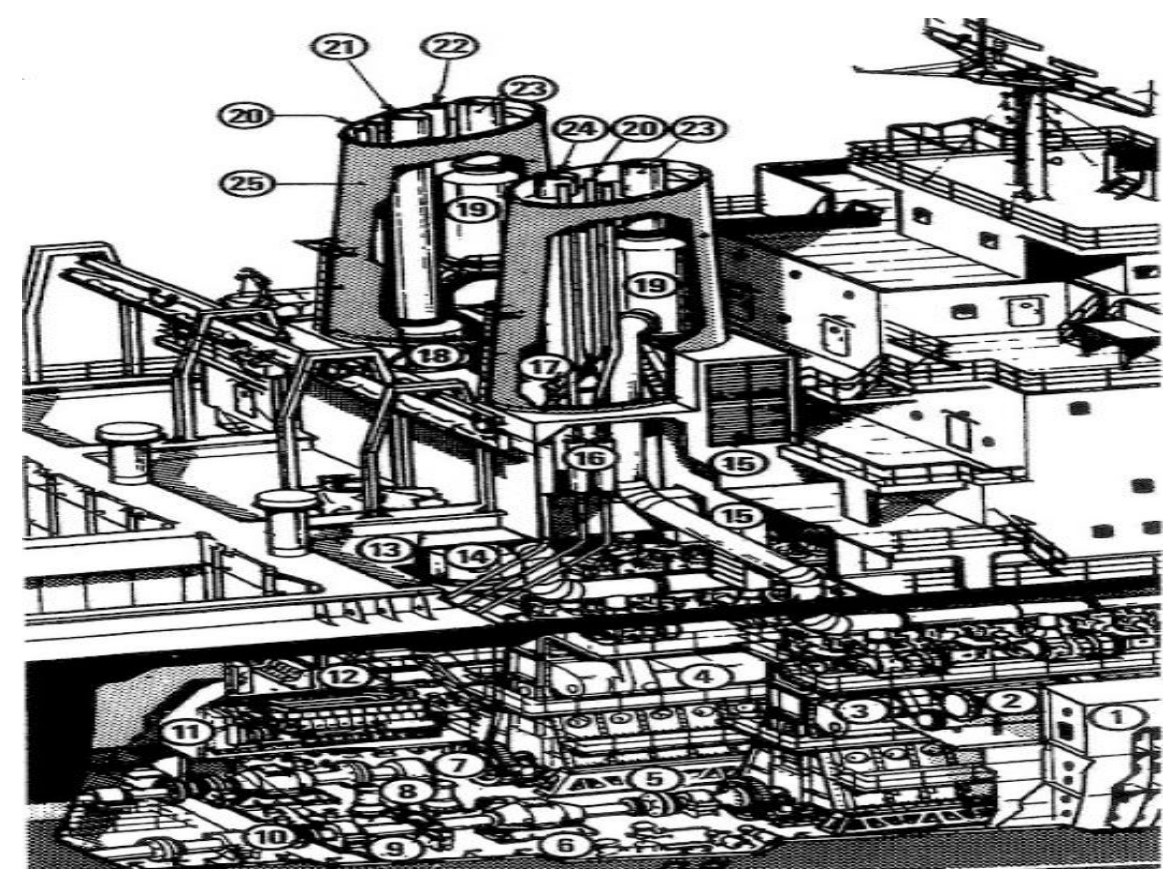

Figure 3: Engine room compartment in general.

Table 2. The engine room compartment in general

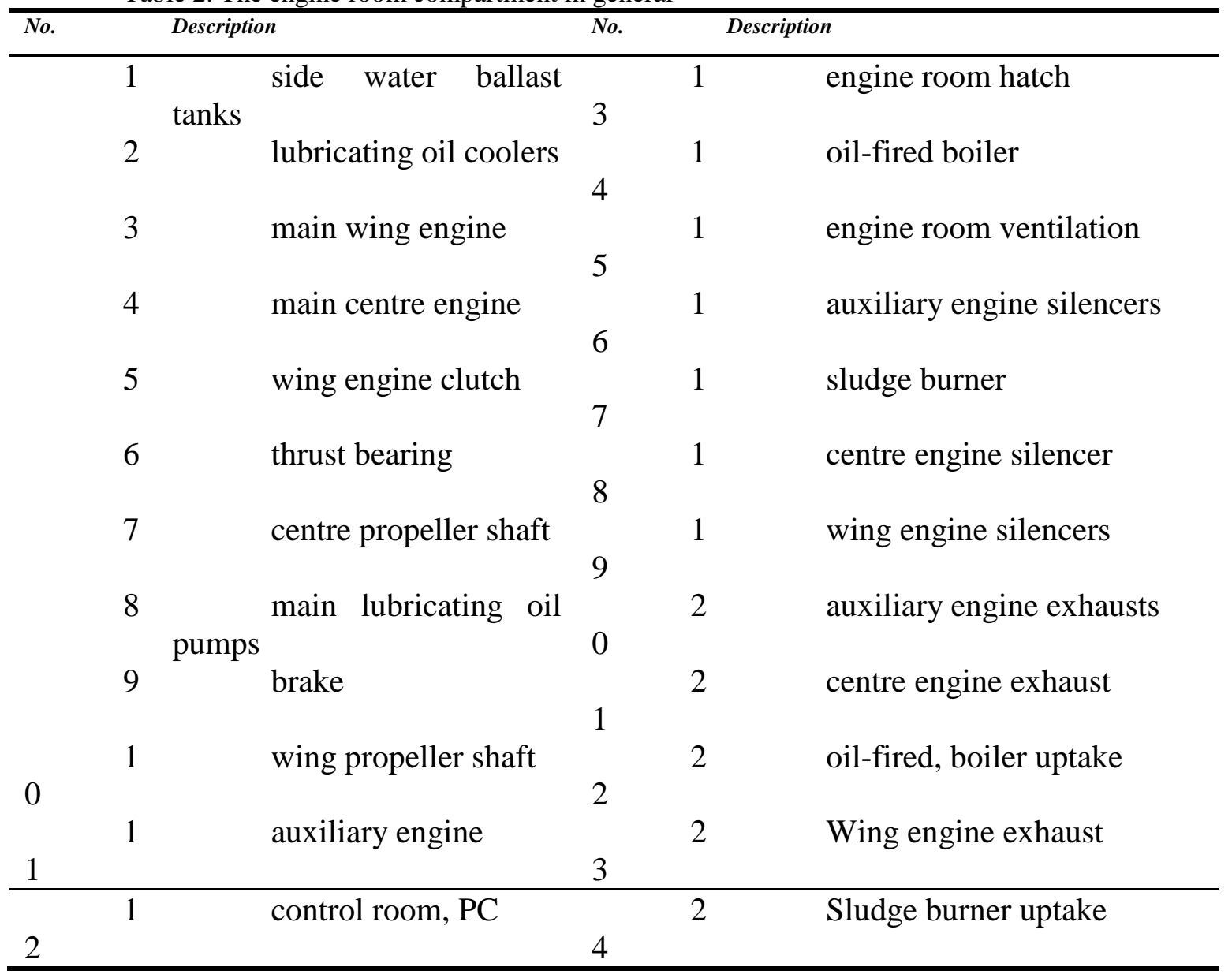




\section{Wheelhouse Devices}

\subsection{Speed log}

Speed log electronic measurement device is measuring the vessel's speed through water the unit measurement is nautical Knot. Many types can be classified by the principle of work the old fashioned is measuring the speed using water pressure, nowadays the most popular is the electromagnetic induction .The speed log plays major role in navigation system (Edward, W., 1937) .The output signals depends on the type and model of the device, some devices output signal is pulses 200 pulse /minute and the common output of the contemporary devices is NMEA 183 or NMEA 2000.

\subsection{Echo sounder}

Echo sounding is a type of SONAR (sound navigation and ranging) used to measure the depth of water under the vessel's keel as review,IHO,standard for hydrographic surveys (2008), by transmitting sound pulses (100pulses/second) into water generates acoustic energy in water, the pulses of sound penetrates the water depth via approximately velocity 1500 meter /seconds. The time interval between emission of the sound and reflecting of a pulse is recorded and translated into meter or feet unit depends on the users demands. In the modern devices the output signal either NMEA183 or NMEA 2000.

\subsection{Navigational telex (navtex)}

NAVTEX (Navigational by Telex) is a maritime safety information provider system, is providing vessel's crew by navigational, meteorological warning and forecasts. The working frequency is automated medium frequency in addition direct-printing service for delivery of navigational and meteorological warnings and forecasts printed, Nowadays the Navtex devices eliminates the direct-printing technology by using the internal storage memory to store the receiving massages from various land stations (Tetley, L. et al., 2001). The common output for interfacing signal is NMEA 183.

\subsection{Global Position System (GPS)}

The Global Positioning System (GPS) is a space-based satellite navigation system that provides location and time information in all weather conditions, anywhere on or near the Earth where there is an unobstructed line of sight to four or more GPS satellite coverage approximately twenty four satellites provide global coverage; four in each of six orbital planes (Sonnenberg,G.J.,1988). Output signal allow system be interfaced is NMEA 183.

\subsection{Automatic Identification System (AIS)}

The automatic identification system (AIS) is a VHF transponder onboard, broadcasting continuously the vessel's position and data .The location and data can be shared with nearby ships and VTS vessel traffic services in post. The marine frequency channel ranges which the AIS handles with it 161.975 and $162.025 \mathrm{MHz}$ the Automatic Identification Systems (AIS) caters for aid in the avoidance of vessel collisions by providing positional data (latitude and longitude) and attributes (such as speed, name, size, type, etc.) of equipped vessels. AIS play an increasingly important role in enhancing the Maritime Domain Awareness of many countries around the world. Key types of AIS data include vessel name ,IMO number ,MMSI maritime mobile service identity number, vessel call sign, vessel type, vessel dimension (length, draft \& beam), ship position (built in GPS), Speed over ground, Course over ground, Heading ( heading signal from gyrocompass) and estimated time arrival(Tetley, L. et al., 2001). Output signal is NMEA 183 and NMEA 2000. 


\subsection{Radar}

Radar is radio detection and ranging, generating electromagnetic wave through magnetron device and podcasting the generated waves via scanner (antenna), the reflection of the magnetic waves is carrying data of other vessels (speed and position comparing with vessel position), bodies, boats, whales or rocks nearby the vessel. Marine radars types are $\mathrm{x}$-band or s-band radar to provide bearing and distance of ships and land targets in vicinity from own ship (radar scanner) for collision avoidance and navigation at sea. According to the SOLAS convention requirements, the vessel should install two radars $\mathrm{S}$ Band radar $(3 \mathrm{GHz}$ frequency) and $\mathrm{X}$ band Radar (9 GHz frequency) each Radar had advantages and disadvantages and both of them generate output signal is video

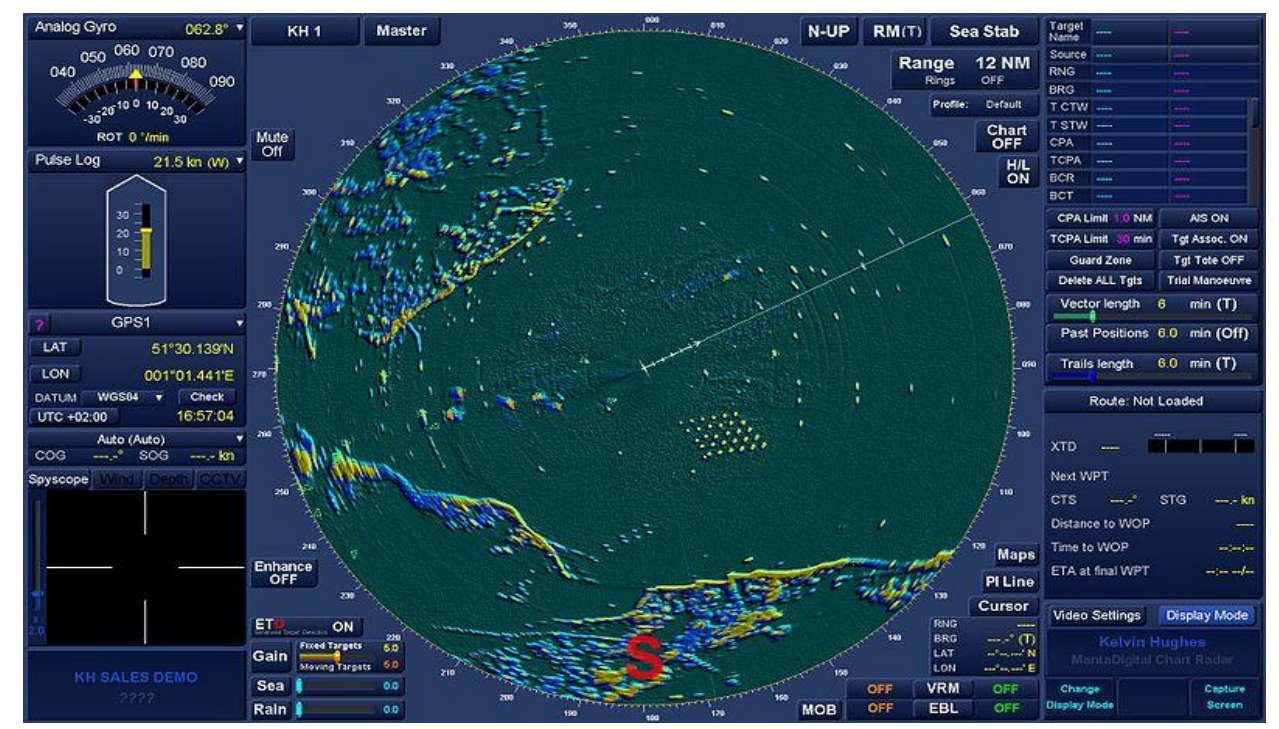

Figure 4.Radar screen view

graphic array (VGA).Via long range monitoring radar screen in the main office the management department can detect any foreign movement heading to the vessel or nearby vessel during sailing. By this feature the vessel crew can be alerted within seconds to take actions and procedures against piracy, terrorism or collisions taking into account the watch keeper absence (Bole, A. et al., 2005)

\subsection{Gyrocompass}

A gyrocompass is similar to a gyroscope. A gyrocompass is used for navigation onboard ships it is a sailor guider. The gyro compass as a sensor ascertains and determines the true north independently from the earth's magnetic fields, thus, permits steering of course referred geographical north. Via electronic boards installed inside the Gyrocompass device are calculating the vessel trends from north and measuring the emergent trends by degrees from $0-360^{\circ}$. Gyrocompasses are widely used on ships .It is the vessel's eyes to find the true north or how much the vessel's tendency about north? It is the high accuracy and alternative for the conventional magnetic compass. Nowadays the magnetic compass is not reliable due to natural magnetic deviation caused by earth in several areas .Albeit magnetic compass's accuracy with limitations nevertheless today magnetic compass used as substitute in case of emergency such as ,losing the power supply or physical damage in the main components of the gyrocompass (Saffors,E.,1070). The output signal from electronic interface card of the gyro compass is NMEA 183.

\subsection{Global maritime distress and safety system (GMDSS)}

Marine vessel Titanic sank due to a collision with an iceberg in the cold ocean , 1500 passengers have perished, fortunately another 700 passengers were saved thankfully to Titanic's two radio officers onboard. They insisted to get help and rescue from nearby vessels by conventional radio signals. Here is the role of the modern GMDSS (Global Maritime 
Distress and Safety System) is specifically designed to automate a ship's radio distress alerting function, and, as a consequence, removes the requirement for manual (ie: human) watch keeping on distress channels by pressing one push button called DSC digital selective call to transmit the distress call to land stations via medium frequency ,high frequency, very high frequency and satellite medium communication are carrying the vessel's information, position and the type of distress(IMO,2013) .The GMDSS system consist of several communication physical devices according to the SOLAS requirements and IMO . The vessel complies with operational requirements according to the region sailing or sea Area; each radio communication device can be covered the sailing region. The new system divides the world's ocean into 4 areas A1, A2, A3 and A4 (Furuno electronic co., 2000). Mainly we are interesting to record and transmit through long range the voice of the marine communication system via VHF devices installed onboard .The output signal form VHF devices is audio signal.

\subsection{Bridge navigational watch alarm system (BNWAS)}

A bridge navigational watch alarm system, abbreviated BNWAS, is an automatic system which sounds an alarm visual and acoustic on the vessel's special areas and general in worst case. If the watch officer on the bridge of a ship falls asleep, becomes otherwise incapacitated, or is absent for too long a time. The BNWAS is automatically engaged when the ship's autopilot is activated (Tetley, L. et al., 2001). The new system embedded of the wheelhouse equipments and it is mandatory. Since the last incidents occurred due to officers or watch keeper's mistakes due to indifference to night watch keeping, the BNWAS system has been created to avoid watch keeper mistakes such as naps night and indifference. The philosophy of works is if the watch keeper (officer) does not moving within five minutes in the wheelhouse area the alarm will be occurred and pre-warning alarm to the master or chief officer cabins to alert that vessel not under command and to take proper action and save the vessel from expected disaster. The officer movement is detecting by motion cameras or by pressing every 5 minutes the reset push button located on the wings of the wheelhouse (BNWAS Basic, 2014).The time setting can be minimum 3 minutes and maximum 15 minutes. The output signal to VDR is binary (on/off) or serial RS232.

\subsection{Ship security alert system (SASS)}

The Ship Security Alert System (SSAS) is part of the ISPS code (International Ship and Port Facility Security Code) and is a system that contributes to the International Maritime Organization's (IMO)'s efforts to strengthen maritime security and prevents and resists acts of terrorism and piracy against shipping. In case the vessel exposed to piracy attacks or terrorism the system is transmitting alert distress over Cospas-Sarsat and to the security officer on land by activating the hidden push button (Performance standards for ship security alert system, 2002). Where is the international Cospas- Sarsat are the satellites based on search and rescue program.

The Philosophy of work is when the vessel is attacking by piracy or terrorism the duty officer press the alert push button to call distress .The digital massage is carrying the vessel name, country code, MMSI (maritime mobile service identity), time and location .The massage is receiving by management office (security company officer) and according to the situation the security procedures will be undertaken. The SASS high level of anti piracy and terrorism security system and is contributing big role in protection the vessel and the crew .let's anticipate other scenario, the vessel under pirate attack and due to physical or panic causes the SASS's push button not triggered, the management will be informed by abductors when they will request ransom there is always weakness.

Koard, J. (2010) mentioned that "When a ship is under pirate attack it can send a distress message using its secret button (officially called the Ship Security Alert System or SSAS). However, a potential weakness of the SSAS regulations is that security alerts are 
required to be routed only to the Company Security Officer and the Flag. This means you had better hope that your company security officer A) has his blackberry on him B) His battery is charged C) he is sober. Otherwise the management of your distress will suffer". Generally the SASS output signal is NMEA 183 or NMEA 2000.Some types can provide RS485 serial communication.

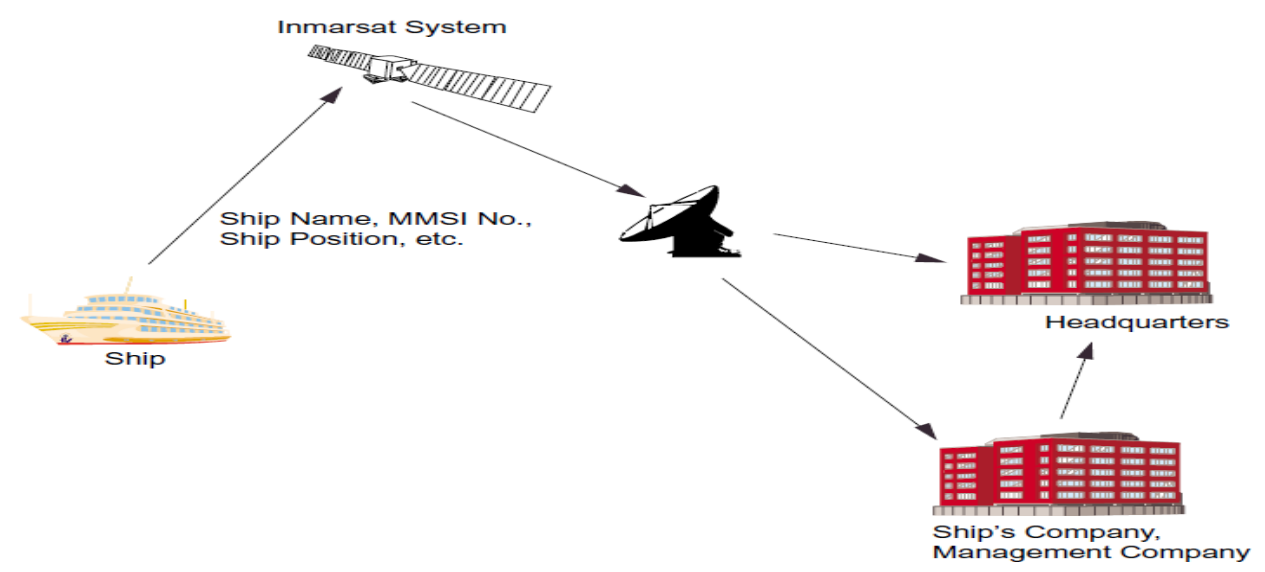

Figure 5. SASS transmission overview

\subsection{Electronic Chart Display \& Information System (ECDIS)}

Since the humane started navigation on the sea and map was in parallel with human starting, the maps formulated into several types depend on sun, stars or landmarks .Nowadays the maps converted into science name called Marine Charts. Without charts the vessel's crew cannot easily determine the destination and the route. The charts need updating periodically. The navigator officer determine the vessel position from the GPS and located on the Chart then will draw the route of the vessel to required destination, it takes time and efforts which may cause overload on the duty officer and dispersion him. Due to innovation and navigation safety and security the inventors submitted new system called (ECDIS) Electronic Chart Display \& Information System is a simple computer-based navigation information system that complies with International Maritime Organization (IMO) regulations and can be used as an alternative to paper nautical or paper charts. By using the ECDIS the position information from position, heading and speed through water reference systems and optionally other navigational sensors are available and showing on the main ECDIS screen twenty four hours .Notwithstanding the new ECDIS system in the vessels paves and contributes to smooth and easy navigation operation in the open sea but in general not feasible if the input signal of the GPS lost due to physical defect, the ECDIS's operation and benefits will be useless. The watch keeper officer will be forced to return to the marine conventional paper maps.

Spenser, C. et al.,(2011) expressed their view as follow "There is the risk with ECDIS that navigators may become dependent on monitoring and that when a sensor fails, the navigator must return to first principles to deal with the situation. This in turn may lead the navigator to incur additional errors in dealing with the situation known as 'knowledge based errors'. Let's conceive that the vessel lost the meaning of the position detection and the vessel route is tracking by main office (long range), the vessel under owner eyes He knows where is the vessel even the crew lost the position due to physical damage or power supply failure .The main offices will supports and guide him to the correct route. The main signal can be transferred to the VDR if required is VGA video signal. 
3.13 Long-range identification and tracking (LRIT)

2014, Vol. 4, No. 3

The long-range identification and tracking (LRIT) of ships was established as an international system on 19 May 2006 by the International Maritime Organization (IMO) as resolution MSC.202 (81). This resolution amends chapter V of the International Convention for the Safety of Life at Sea (SOLAS), regulation 19-1 and binds all governments which have contracted to the IMO .An overview of the operational concept of the LRIT, LRIT is a maritime domain awareness (MDA) initiative to enhance maritime safety, security and protect the marine environment. LRIT allows Member States to receive position reports from vessels operating under their flag, vessels seeking entry to a port within their territory, or vessels operating in proximity to the State's coastline (Peachey, G. 2011) .

For more practical clarification please visit site tracking vessels of the ABM fleet: http://www.satpro.org/kundenlinks/concilium.php

Frankly I have contributed in the establishment of this service for Arab bridge maritime company fleet. And we are proud that we are the fist marine company (ABM) in the East implemented this private system in 2010.Monitoring the ABM fleets position from long range distance.Inspite of the vessels all the time under monitoring and watching from long range but the information that we are receiving not enough to achieve the high level of long range security and safety. In Contrast it was in 2010 new era of monitoring your fleet from long distance.

\subsection{Voyage Data Recorder (VDR)}

VDR is the voyage data recorder it is Similar to the black box carried on aircraft, marine VDRs enable accident investigators to review procedures, instructions and communications in the moments before an incident and help to identify the cause of any accident ,helps to analyze the accidents and mistakes and determine the responsibilities. The VDR combines data from the sensors onboard and stores it in an externally mounted Crash Survivable Module (CSM), so the investigator can re- scenario the events via recorded evidence. The CSM is a tamperproof unit designed to withstand the extreme shock, impact, pressure and heat, which could be associated with a maritime incident .To this day the CSM cab be in two forms 1) fixed capsule 2) floating capsule. The CSM may be retrieved from the vessel and the stored data replayed by the authorities for investigative purposes (Kelvin Huges com.2011).

As mentioned about VDR that is our aim to find reliable solution to integrate navigation sensors and main engine's safety sensor with exploiting the existing and installed devices onboard to reduce the project cost into one interface unit .In this part VDR is the most significant and center of aggregated navigation and vital signals from both fundamentals wheelhouse compartment and engine room section (will explain later the main devices in the engine room).The VDR devices can interface with all navigation signals via physical communication wires and the data can be transferred to the VDR in form as RS232, RS484 ,NMEA2000 and NMEA 183 .The VDR investors are taking into account the old model of devices and how interfaces to the old models by converting the signals into serial communication to feed the VDR and recording system. For instance some old versions of the speed log which measuring the speed their output signals are pulses/mile (200 pulses /mile) the VDR companies cured this requirements by installing physical internal converters. The required data to be recorded according to the SOLAS requirements and the marine rules as the following:

Table 3. The VDR devices \& signals

\begin{tabular}{|l|l|c|c|}
\hline No. & Device & Description & \multicolumn{3}{|c|}{ Output Signal type } \\
\hline & GPS & date and time & 2000 \\
\hline & Log speed & Speed \& distance & \multicolumn{3}{|c|}{ Pulses or NMEA } \\
\hline
\end{tabular}




\begin{tabular}{|c|c|c|c|}
\hline & Gyrocompass & Heading (North) & NMEA183 or2000 \\
\hline & Radar & Post display image & VGA \\
\hline & Anemometer & Wind speed direction & NMEA 183 \\
\hline & Echo sounder & Depth under keel & NMEA 183 \\
\hline & Bridge audio & $\begin{array}{l}\text { Mic - internal and } \\
\text { external }\end{array}$ & $\begin{array}{c}\text { Audio signal } \\
\text { connect to multiplexer }\end{array}$ \\
\hline & BNWAS & Watch alert & NMEA 183 \\
\hline & VHF & Communication & Audio \\
\hline 0 & Hull & Opening doors & Binary to Serial \\
\hline 1 & Rudder & Steering - status & Serial RS232 \\
\hline 2 & Fire system & Fire \& smoke detection & Binary or RS232 \\
\hline 2 & Autopilot & Setting and status & NMEA183 \\
\hline 3 & Engine /propeller & Order and response & RS232 or $4-20 \mathrm{~mA}$ \\
\hline 4 & $\begin{array}{llll}\text { doors } & \text { Water and fire } \\
\end{array}$ & Indication and control & $\begin{array}{lrrr}\text { BS232 } & \text { Binary to serial } \\
\text { RS }\end{array}$ \\
\hline 5 & Main engine & $\begin{array}{l}\text { Alarms and safety shut } \\
\text { down }\end{array}$ & RS232 or RS 485 \\
\hline 6 & Water leakage & $\begin{array}{l}\text { Detection water inside } \\
\text { the vessel }\end{array}$ & Binary to RS 232 \\
\hline
\end{tabular}

\section{Main Engine Devices}

In this paper will be demonstrated the most vital and significant safety components located in engine room. The engine room is the power and muscles of the vessel .The propeller shafts of the vessel are powered by diesel engines stem engines or gas turbine engines whatever the way for generating the power for propulsions, we need to control and protect engines from abnormal operation and failures.

The vessel's electricity is generating via diesel generators, steam generators, hydraulic generators or gas turbine generators. Also same principle safety must be applied and perused

\subsection{Main engine safety system}

Main engine safety devices had many definitions I prefer the definition depend on experience The main engine safety is devices are installed in the system to safe guard the main engine in case of any abnormal effect in the system such as low oil pressure, over speed main engine ,crank case high pressure, oil mist detector in crank case, jacket cooling water temperature, high exhaust temperature and turning gear interlock ,by other words physical main engine protection system to avoid big damage and big cost in daily operation ( Hall, D.T.1999)

The signal output from the above mentioned categories depends on the main sensors. The type of the sensors usually analog signal or binary (on /off). Whatever the kind of the sensor eventually will be connected to the PLC (programmable logic controller) or controller logic systems.All these signals will be sent from controller to the VDR as one signal to be recorded in the VDR to show the status and behaviour of the main engine (Jackson,L.2006).

\subsection{Diesel generator safety system}


The diesel generator safety devices depends on the prime mover type .Will mention the general safety such as over speed, low oil pressure, high temperature cooling water and electrical protection devices such as reverse power system (Hall, D.T.1999).The output signals same the main engine sensors analog and binary depends on the senor type .Eventually all are aggregating in controller system or PLC then transfer into one signal to VDR via serial communication.

\subsection{Tanks Level}

The tank levels are very important to monitor and control due to the major role they are playing in the vessel stability system. the level tanks are measuring by analog sensor and the output signals the common output signals are 4-20mA or resistive amount is measuring by Ohm (Hall, D.T.1999).All sensor are collect into one system level tanks control processor and can use additional converted to convert the $4-20 \mathrm{~mA}$ signals into serial communication transfer to the VDR .

\subsection{Bilge Alarm Level}

The bilge system definition is the part of the underwater body of a ship between the flat or bulkhead of the bottom and the vertical topsides, usually aggregated of the oil, fuel or water leakages and (Hall, D.T.1999).

Necessarily to monitor and watch the level to avoid the flooding which may cause damage to the engine machineries especially electrical machinery. More over the liquid in the bilge system is contaminated liquid therefore it is forbidden by maritime law to drop or pump the contaminated liquid to the clean sea. Seriously according to the maritime law is considered as crime.

\subsection{Fire extinguish safety system}

In the vessel's extinguishing systems are various form vessels to another vessel depend of the vessel's type and model and the marine rules. Generally in the main engine the fire extinguishing via $\mathrm{CO} 2$ gas system. In the RORO vessel's main garage the fire extinguishing via Sprinkler system, where is the system consisting of a network of overhead pipes that release water automatically when a predetermined temperature has been reached in the main garage or passenger saloon. Some types of fire extinguishing system via fog system.

Hi Fog system is a water mist system is a fire protection system which uses very fine water sprays (i.e. water mist). The small water droplets allow the water mist to control, suppress or extinguish fires (Hall, D.T., 1999).

The main fire extinguishing systems are controlled by electrical control systems .Often the feedback signals are binary can be converted into serial communication and send to the VDR recording system.

\section{Principle of the Integration}

The principle of integration is collecting all aggregated signals from mandatory devices into one station, this station able to contribute and share information via the artificial network system onboard. Thus aggregated signals will be transferred to land station or main office as one packet. As above explanation of each device onboard and type of the output signal we can recognize that all signals can be handled easier with VDR and more reliable, because our scope is make integration system with minimal cost and reliable with trade on the available components onboard.

As sheet (No.1) illustrated the physical layout of integration for each device either controlling ,sensors or communication voice. After integration the system will prepare real time massages to send through external communication satellite to the land office or whatever Palm 
of a hand platform. Many companies like Kelvin huges seeks to achieve pilotage in the palm of a hand like unmanned drones. The VDR voyage data recorder equipped with interfacing electronic input cards to receive, translate and analyze the signals sentences. The sentences form various electronic components or the summations of the various signals will be formulated into one stream of data and recorded inside the VDR's storage area as shelves.

\subsection{Main engine integration devices}

For integration and summation a huge amount of sensors like a feedback, actuators and control sensors we need reliable and fast aggregating programmable center. As above mentioned the main engine compartment sensors consist of various types such as analog, binary, inputs and outputs to collect all signals in one system can handle and deal with it via PLC (programming logic controller).

\subsubsection{Programmable logic controller (PLC)}

Control engineering has developed over time. In the past humans was the main method for controlling a system, monitoring and issuing the decisions. More recently electricity has been used for control and early electrical control was based on conventional control relay units which are losing the precision and reliability and caused aging electricity connection and interference noise. These relays control power to be switched on and off without a mechanical switch or manual method. It is common to use relays to make simple logical control decisions. The development of low cost computer has brought and created the most recent revolution, the Programmable Logic Controller (PLC). The appearance of the PLC began in the 1970s, and has become the most common choice for manufacturing automation controls (Salhat, M. et al., 2005). Programmable Logic Controllers (PLCs), also referred to as programmable controllers are in the computer family. They are used in commercial and industrial applications. A PLC monitors Inputs, makes decisions based on its program, and controls

Outputs to automate a process or machine, nowadays the PLC is necessarily for all industrial field, aviation field, medical field commercial filed and marine field (Jack, H.2007).

\subsubsection{Input output sensors}

The PLC's efficiency of work depends on the sensors without outsourcing or feedbacks the PLC is blind .The sensor generates signals according to ambient sensing and the PLC convert these signals into logical language. The signals in the automation world are various and huge (Salhat, M. et al., 2005). A sensor is a device that converts a physical condition into an Electrical signal for use by the PLC. A discrete input also referred to as a digital input, is an input that is either in an ON or OFF condition. Pushbuttons, toggle Switches, limit switches, proximity switches, and contact. An analog input is an input signal that has a continuous signal. Typical analog inputs may vary from 0 to 20 milliamps, 4 to 20 milliamps, or 0 to 10 volts. A discrete output is an output that is either in an ON or OFF condition. Solenoids, contactor coils, and lamps are examples of actuator devices connected to discrete outputs. An analog output is an output signal that has a continuous signal. The output may be as simple as a 0-10 VDC level that drives an analog meter (Salhat, M. et al., 2005).

\subsubsection{Programming}

A program consists of one or more instructions that accomplish a task. Programming a PLC is simply constructing a set of Instructions. There are several ways to look at a program such As ladder logic, statement lists, or (FBD) function block diagrams (Jack, H., 2007). The PLC scan process is working during four important stages first stage reading the inputs form external sensors, orders or feedbacks .PLC is converting the input analog or digital signals such as temperature, speed, levels or density into symmetrical numbers (computer language 1, 0, 1, 
1).Second stage execute program, the program which is written by designer or programmer is including analytical blocks program for each input the written program responsible for handling and connecting between inputs and the customer operation behavior. Third stage is diagnostics and communication . The last stage is update output, the program will handle with external PLC environment by OUTPUTS ,the outputs can be analog such as 0-10 V,4-20 mA ,Contacts on /off or Serial communication output .Main scope here in this paper to collect all main engine data from various signals into one reliable PLC system and converted into one output serial communication like RS232, RS485 or Ethernet cable (for 100 meter )carrying data and the VDR in the wheelhouse can deal with output serial communication and record them .

\subsubsection{Supervisory control \& data acquisition (SCADA)}

SCADA is an abbreviation for supervisory control and data acquisition. SCADA systems are used to monitor and control a plant or equipment in industries such as telecommunications, water and waste control, energy, oil and gas refining and Transportation and our scope here to monitor the vessel data in the office by SCADA system. Encompass to integrate data acquisition systems with data transmission systems and HMI software to provide centralized monitor ring and control system for numerous process inputs and outputs. SCADA systems consist of:

a) One or more field data interface device usually RTU (remote terminals unit ) or PLC with interface field like main engine sensors and others as above mentioned in paragraph 4.

b) A communications system used to transfer data between field data interface devices (wheelhouse devices), control units (main engine sensors) and the computers in the SCADA central host (main office on land). The system communication can be radio, telephone, cable, satellite, etc., or any combination of these. We will work on satellite system descried on section 7 .

c) A central host computer server or servers (sometimes called a SCADA Center or master station, we will call it land station.

d) Custom software \{sometimes called Human Machine Interface (HMI) software or Man Machine Interface (MMI) software s systems used to provide the SCADA central host and operator terminal application, support the communications system, and monitor and control remotely located field data interface devices the popular software Wincc from Siemens, Review by Allen Bradley .

Why our choice here is the SCADA in the office station because the SCADA focuses on supervisory level with minimum hardware and software cost.SCADA system enable to receive the information from sailing vessel and translated into multi screens with readable data and understandable .
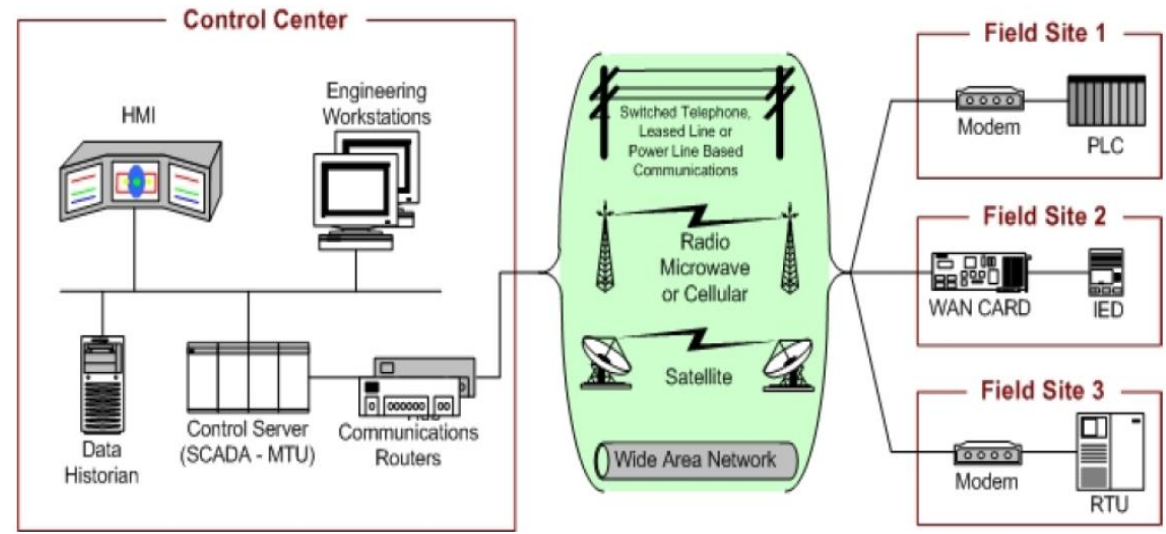
Figure 6. SCADA system general layout.

\section{Network Technology in the ship}

The simple computer network is consisting of two connected computers or more for more users.Ecah computer is consisting the network system via NIC network interface card which is linking computer to the external world of network the computers communicating and sending bits through physical medium connection such as telephone wires, coaxial cables or radio signals in case wireless method communication .In our conventional world in a moment of the rush hours is necessitating traffic police man to arrange an coordinates the car traffics ,so in small network system also necessitated traffic police man but we called Hub or switch .To communicate with other world the global network of networks the internet, the router help you as simple network to communicate with other networks (Laudon,K.C. et al.,2014).

Here we will concentrate on the LAN local area network on the vessel between main engines's PLC or SCADA system and the wheel house electronic components to VDR. The simple network was chosen to disciple the network in common merchant vessels, for more information some merchant vessels uses more sophisticated network systems which are requiring corporate Servers uses in the cruise vessels floating villages.

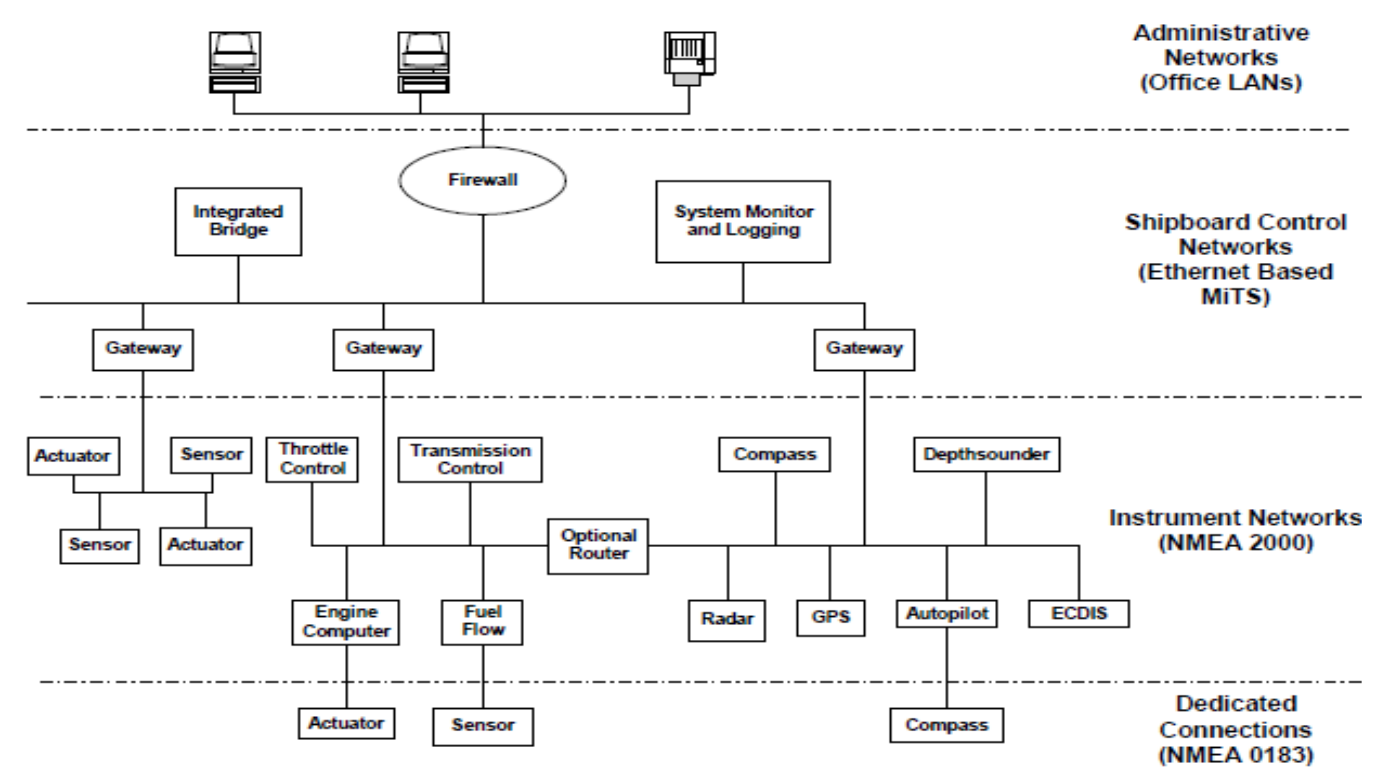

Figure 7.Shipboard network and interfaces overview

\subsection{NMEA 183}

NMEA stands for National Marine Electronics Association (of the USA). NMEA183 was established in 1983 as a voluntary industry standard for data communications among shipboard electronic devices. Simple ASCII it uses, serial communications protocol that defines how data is transmitted in a "sentence" from one 'talker' to one or more 'listeners' at a time, and therefore cannot be used to create networks. Data transmission is slow by today's standard at 4800 bits / second and the standard does not allow for multiple 'talkers'. However it is still in widespread use and is perfectly adequate for situations where one piece of equipment, for example a hand-held GPS, is to be connected to another such as an on-board chart plotter where the user wishes to integrate the two sets of data (Maral,G. et al.,2002).

Table 4.Parameter of the serial interface 


\begin{tabular}{|l|l|l|}
\hline No. & Description & Parameters \\
\hline 1 & Baud rate & 4800 \\
\hline 2 & $\begin{array}{l}\text { Number of data } \\
\text { bit }\end{array}$ & 8 (bit 7 is 0) \\
\hline 3 & Stop bits & 1 or more \\
\hline 4 & Handshake & None \\
\hline 5 & Baud rate & None \\
\hline
\end{tabular}

\subsection{NMEA 2000}

Currently the accepted standard across the international marine industry, NMEA 2000 is much more sophisticated than NMEA 0183 in that it allows multiple units to simultaneously both transmit and receive data. With the inclusion of multifunction displays into a networked system the user can then choose any combination of data outputs to be displayed at any position or for any situation. It is NMEA 2000 that has made possible the development of the integrated navigation and control systems that are now being fitted on craft of almost every size and application (National marine electronic association, 2002). Physical nodes: Up to 50 connections Functional nodes: Up to 254 network addresses. Length: Up to 200 meters (at $250 \mathrm{kbits} / \mathrm{second}$ bit rate).

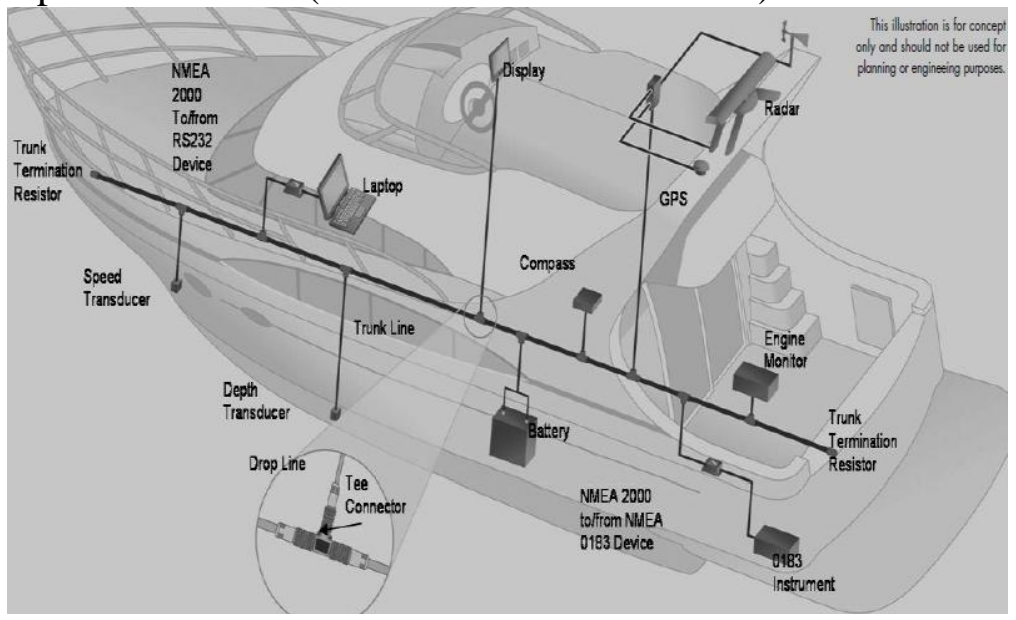

Figure 8. NMEA 2000 network sample.

\subsection{Ethernet}

Ethernet LAN topology is currently the most common network architecture. Ethernet is a widely-used cable-based technology for transmitting very large amounts of electronic data between units of equipment within a LAN (local area network) and as such can be found in all forms of computing technology across every aspect of modern life (100 meter). Capable of transmitting at rates of $10 \mathrm{MB}$ per second and more (versus $0.25 \mathrm{MB} p / \mathrm{s}$ for NMEA 2000) it can play a valuable role with marine electronics that process high volumes of data, for example radar, electronic charts and weather overlay information, and it is now common to find such units that now offer both Ethernet and NMEA 2000 connectivity.

Table 5.Talker Identifiers samples

\begin{tabular}{|l|l|}
\hline EC & $\begin{array}{l}\text { Electronic Chart Display \& Information System } \\
\text { (ECDIS) }\end{array}$ \\
\hline ER & Engine Room Monitoring Systems \\
\hline RA & RADAR and/or ARPA \\
\hline SD & Sounder ,Depth \\
\hline
\end{tabular}




\begin{tabular}{|l|l|}
\hline VD & Velocity sensor Doppler \\
\hline GP & Global position GPS \\
\hline ZA & Timekeeper -automatic clock \\
\hline AG & Autopilot -general \\
\hline
\end{tabular}

All three technologies continue to play important roles in the inter-connecting of marine electronic devices; the selection between three technologies depends on the user demands and the kind of the operation.

\section{Concept of Satellite Communication}

The satellite communications means communication through satellite. The advent of earth's artificial satellite has extended the range of line-of- sight propagation paths and made possible for transmission signals for far ways through oceans. In the maritime community, satellite communication systems such as Inmarsat provide good communication links to ships at sea. These links use a VSAT type device to connect to geosynchronous satellites, which in turn link the ship to a land based point of presence to the respective nation's telecommunications system.Nowdays many SAT companies is offering broadband communication via many various services such as mini- VSAT broadband ,Inmarsat fleet,Inmarsat fleet broadband or Intelsat broadband The satellite communication technology initially $265 \mathrm{~Kb}$ speed download and upload $128 \mathrm{~Kb}$ in maritime field nowadays by new broadband communication technology we can achieve speed up to $50 \mathrm{Mb}$ download and upload .

\subsection{Some of the Popular Satellites Servicing Marine Field}

Some popular Satellite in modern and broadband marine communications. The choice of the communication satellite depends on the customer users and owner budget .Long range security and safety system requiring medium speed of downloading and uploading to view vessel data of navigation security and main engine safety in real time.
a) INMARSTAT
b) V SAT
c) DSAT
d) INTELSAT

Broadband satellite service is an emerging service which has caught the fancy of many for meeting the demand of worldwide fiber like access to telecommunications services such as computer networking, broadband Internet access, and interactive multimedia and high quality voice. These systems use advanced satellite technology at $\mathrm{Ka}$ band or $\mathrm{Ku}$ band frequencies to achieve the high bandwidth requirements (Maral, G. et al., 2002).

\section{Conclusion Outlook Future}

This paper has been described the principle method of long range monitoring and control the security / safety system via integration marine electronic components of the wheel house and main engine department by exploiting and using the installed devices onboard without incremental improvement or refurbishment of navigation or safety components, Sending the integrated data of the vessel via marine satellite communication broadband technology through oceans to main office, which is in our opinion is the extra cost in the communication 
system (download/upload) service .Eventually the vessel data can be monitored from long range through oceans with real time on SCADA system or similar in main office. The monitoring and control from long range will emerge many benefits for the crew vessel ,owners and vessel itself .Managing, controlling and protecting will increase and enhance the vessel performance and activity in other side will be safely sailed this paper tried to simply explain how is the system to integrate to achieve the requirement goals of tracking from long range.In the Outlook future Electronic integrated bridge concepts are driving future navigation system planning. Some of marine companies purses to achieve the vessel navigating from long range by Palm of a hand. The communication technology exponentially developed in last ten years which is Emphasizing that there is the development of more and more speed in the world of information transfer and synchronizing. Access to the moon and landing Speech over the air See the picture through the Box All were dreams and precisely achieved.

\section{Acknowledgments}

I want to admit that without the practical experience at sea I couldn't write this report. And I thank all the references that helped me to coordinate my ideas and arrange. And special thanks to Dr. Evon Abu Taieh for encourage and motivate us to write and express what inside of us of knowledge, her continuous assistance and support always will be strongly appreciated. Will not miss of my memory to thank associate Prof. Dr. Firas Rewashdeh Who taught me the foundations of scientific research and principles.

\section{References}

Brookes, B. (2013) Deputy President IET, Global challenges in maritime securities .Available from www.theiet.org/transport. (Accessed, 14 May 2014).

Bole, A., Dineley, B., \& Wall, A. (2005). Radar and ARPA manual. Second edition, 2005.

BNWAS Basic. Bridge navigational watch alarm system. (2014).SAM electronic GmbH. L3 Communication Company. Available from www.sam-electronics.de .Germany ; accessed 24 May 2014).

Edward, W. (1973). A History of Marine Navigation, G. T. Foulis \& Co. Ltd., Henley-on-Thames. Oxfordshire.

Furuno electric co. LTD. (2000). Catalogue No. GC-019h, GMDSS guide, printed in Japan.

Hall, D.T. (1999). Practical marine electrical knowledge. Second edition, Witherby, London.

IHO Standards for Hydrographic Surveys. (2008), published by the International Hydrographic Bureau. (5th Edition). Special publication no.44.February 2008.

IMO, GMDSS global maritime distress and safety system. (2013) edition.IMO publishing, United Kingdom.

Jackson, L. (2006).Extra first class engineer's certificate .Reed's marine engineering series. Instrumentation and control systems.

Jack, H. (2007). Automating manufacturing system with PLC's, Version 5, Copyright (c) 1993-2007 Hugh Jack (jackh@gvsu.edu). 
Kelvin Huges com. (2013) .Manta digital voyage data recorder.

Koard, J. (2010). SSAS- what happen when you push the secret button?. Available from http://gcaptain.com/ssas-push-secret-button/;(accessed16 May 2014).

Laudon, K.C., \&Laudon, J.P. (2014). Management information system. Managing the digital firm, 13th edition, Pearson, London.

Maral, G., \& Bousquet, M. (2002). Satellite Communications Systems, Wiley.

National marine electronic association. (2002). NMEA 0183.Standard for Interfacing Marine Electronic Devices. Version 3.01, USA.

Peachey, G. (2011). Chief executive officer. Long range identification and tracking .Australian government.

Performance standards for a ship security alert system. (2002). International maritime organization.IMO.Annex5.Resolution MSC.136 (76).http://www.imo.org/,11december 2002, (Accessed 18May, 2014).

Safford, E. (1979) .Handbook of marine electronic \& electrical systems. First edition.

Sonnenberg, G. J. (1988). Radar and electronic navigation, sixth edition.

Slahat, M., Al-hjazeen, Z., \& Soubeh, K. (2005). Programmable logic controllers, Al-Mujtamaa Al-Arabi, Amman.

Spenser, C., \& Tilsely, D. (2011). Standard safety ECDIS-understanding the future of navigation special edition. Charles Taylor \& Co. Limited. London.

Tetley, L. \& Calcutt, D. (2001). Electronic navigation systems, 3rdedition, Butterworth-Heinemann, Boston. 
